\title{
El Mercurio y la contaminación por actividad extractiva
}

\section{Mercury and the contamination from extraction activity}

\section{Mercure et contamination dans les activités d'extraction minière}

\section{O Mercúrio e a contaminação pela atividade extrativa}

José Alejandro Martínez * Alberto Uribe **

Fecha de recepción: 15 de abril de 2015

Fecha de aprobación: 10 de junio de 2015 PP. 55-76.

* Ph.D (c). Administración Estratégica (PUCP - MSM, Holanda). Magíster en Ingeniería Ambiental e Ingeniero Químico, Universidad Nacional de Colombia. Coordinador del programa de Especialización en Gestión de Residuos Sólidos, Director de la línea de investigación en gestión de residuos, docente e investigador, Universidad EAN.

** Ph.D. y Master of Science en Ingeniería Ambiental, Universidad de Cincinnati (EEUU). Investigador y consultor en el área de agua potable y saneamiento básico, con énfasis en ingeniería de remediación. Docente titular de la Facultad de Ingeniería, Universidad EAN. 


\section{RESUMEN}

Desde mucho antes de la "Locomotora Minera" del Plan Nacional de Desarrollo 2010-2014 de Colombia, las actividades vinculadas a la extracción de minerales han sido fuente de riqueza y problemas en diferentes partes del país. El presente artículo, recoge resultados sobre la investigación que desde 2014 hace la Universidad EAN para consolidar conocimiento sobre la situación de contaminación de suelos con Mercurio ocasionada por los procesos mal desarrollados de extracción de oro en Colombia (Martínez \& Uribe, 2014), buscando contribuir a la identificación de las soluciones para la recuperación de la matriz suelo contaminado con Mercurio.

\section{Palabras clave}

Minería, suelos, recuperación, mercurio, contaminación, tratamientos.

\section{Abstract}

Since well before the mining locomotive from the previous National Development Plan, all the activities related to mineral extraction have been a source of wealth and problems in different parts of the country. This article compiles the results of the research that the EAN University has been performing since 2014, in order to consolidate knowledge about the remediation of soil that has been contaminated with mercury as a result of the poorly developed gold extraction processes, seeking to contribute to the identification of solutions for the recovery of the mercury-contaminated soil matrix.

\section{KEY WORDS}

Mining, soil, recovery, mercury, pollution, treatments. 


\section{RÉSUMÉ}

Avant que ne se produise le récent essor économique de I'industrie de l'extraction minière que défend le Plan National de Développement, les activités ayant trait à ce secteur d'activité ont été une source de richesse mais également de problèmes dans différentes régions colombiennes. Cet article recueille les résultats de recherches réalisées par l'Université Nationale de Colombie depuis 2014 pour synthétiser les connaissances sur les remèdes à apporter aux sols pollués au mercure résultant des processus d'extraction orifère mal exécutés et tente de contribuer à l'identification de solutions pour la récupération de ces sols gravement contaminés.

\section{MOTS CLEFS}

Extraction minière, sols, récupération, pollution, traitements

\section{RESUMO}

Desde muito antes da $<<$ Locomotiva mineira $>$ do anterior Plano Nacional de Desenvolvimento, as atividades vinculadas a extração de minerais tem sido fonte de riqueza e de problemas em diferentes partes do país. O presente artigo recolhe resultados sobre a pesquisa científica, que desde 2014 a Universidade EAN faz, para consolidar conhecimento sobre a remediação dos solos contaminados com mercúrio, fruto dos processos mal desenvolvidos de extração do ouro, procurando contribuir a identificação das soluções para a recuperação da matriz solo contaminada com mercúrio.

\section{PalaVRas-ChaVe}

Mineração, solos, recuperação, mercúrio, contaminação, tratamentos. 


\section{Introducción}

I Mercurio, conocido antiguamente como Hidrargirio ${ }^{1}$ de donde se deriva su símbolo químico $\mathrm{Hg}$, es un elemento químico, número atómico 80 , metálico, que en condiciones de presión y temperatura estándar se encuentra en estado líquido. El Mercurio de origen natural, proviene de la desgasificación de la corteza terrestre, volcanes y evaporación de los océanos (Henry, 2000).

Desde el punto de vista industrial, tiene una gran variedad de usos en sectores tales como la medicina, odontología, fabricación de baterías, insumos científicos y aplicaciones militares. La combustión de combustibles fósiles y la incineración de residuos, comprenden un alto porcentaje de las fuentes antropogénicas de Mercurio. Más de la mitad del Mercurio es utilizado industrialmente y se concentra en el proceso de electrólisis del cloruro de sodio (proceso cloralcali); la industria de pulpa de madera, pinturas y su uso en equipos electrónicos. El hecho de que puede alearse fácilmente con otros metales, particularmente oro y plata -proceso conocido como amalgamación- ha hecho que este material sea muy usado en la industria minera, en particular en la minería artesanal, siendo un contribuyente significativo de contaminación de suelos, sedimentos, aguas subterráneas y superficiales (Boening, 1999).

El Mercurio, dentro de la industria minera artesanal y semiindustrial, es utilizado al aprovechar su propiedad de aleación con metales para lograr la extracción del metal precioso de la veta rocosa. Este disuelve el metal, y la amalgama resultante, es sometida a procesos de separación, calentándola. El Mercurio

\footnotetext{
${ }^{1}$ El vocablo proviene, a su vez, del griego antiguo hydrargyros (hydros = agua y argyros $=$ plata $)$
} 
se evapora, se recupera con un sistema de condensación y el remanente es el metal precioso. El sistema es relativamente sencillo y muy eficiente; sin embargo, durante el proceso siempre hay un nivel de desperdicio tanto en el transcurso de amalgamación, como en el de separación y condensación donde el Mercurio se pierde ya sea de forma líquida o de vapor, liberándose en el ambiente.

Desafortunadamente, se trata de una sustancia tóxica y los efectos en la salud dependen de seis factores principales:

- Forma química del Mercurio.

- Dosis.

- Edad de la persona expuesta -los fetos son los más susceptibles-.

- Duración de la exposición.

- Vía de exposición -inhalación, ingestión, contacto dérmico-.

- Estado de salud de la persona expuesta.

La naturaleza y sus condiciones meteorológicas generan situaciones dinámicas en donde los equilibrios de las especies químicas se pueden romper fácilmente; cuando se analiza la matriz suelo, tal y como se menciona en Wang, et al. (2015), las principales barreras para mejorar la productividad de la tierra en el mundo actual, son la salinización de los suelos y la contaminación de metales pesados del suelo; por ello, en los últimos años se han estudiado las interrelaciones de metales pesados y matrices agua-suelo, así como múltiples mecanismos para la remediación de suelos contaminados por metales pesados (She, et al., 2014). El trabajo que se viene realizando en la Universidad EAN, busca poder reconocer la dinámica del Mercurio que llegó a matrices agua-suelo y desde allí, empezar a abordar posibles alternativas para el desarrollo de la remediación del mismo. De esta forma, reducir el riesgo

\footnotetext{
${ }^{2}$ Para ampliar esta información, consultar: http://www.epa.gov/mercury/effects.htm
} 
asociado a este elemento con relación a las especies vías y a los seres humanos que habitan en zonas contaminadas por la actividad de minería de oro.

\section{El Mercurio y sus formas
químicas}

a forma química en que se encuentra el Mercurio, define los efectos específicos que se pueden esperar sobre la salud humana. Desde este punto de vista, se puede encontrar en alguna de tres formas químicas principales: Metilmercurio, Mercurio elemental y otros compuestos de Mercurio tanto orgánicos como inorgánicos.

Metilmercurio es la abreviación para el catión monometilmercurio (II); esta molécula se compone de un grupo metilo $\left(\mathrm{CH}_{3}{ }^{-}\right)$unido a un ión mercúrico $\left(\mathrm{Hg}_{2}{ }^{+}\right)$dando como resultado el compuesto de fórmula $\mathrm{CH}_{3} \mathrm{Hg}^{+}$. Dado que es un ión positivo, este se combina fácilmente con aniones tales como cloruros $\left(\mathrm{Cl}^{-}\right)$, hidroxilo $\left(\mathrm{OH}^{-}\right)$y nitratos $\left(\mathrm{NO}_{3}^{-}\right)$. También muestra alta afinidad con aniones que contienen azufre, en particular los grupos tiol (-SH) en el aminoácido cisteína y por ende, en proteínas que contengan cisteína formando enlaces covalentes. Esta última condición, hace que el metilmercurio sea fácilmente absorbido por las mucosas gastrointestinales al encontrarse generalmente asociado a cisteína libre u otras proteínas y péptidos que contengan este aminoácido. El complejo cisteinil-metilmercurico, se asemeja a la metionina y a raíz de esta semejanza el compuesto se transporta fácilmente a través de la barrera sangre-cerebro y la placenta. El efecto principal se manifiesta sobre el sistema nervioso 
central, causando síntomas tales como parestésias, pérdida de coordinación física, dificultades al hablar, estrechamiento del campo visual, impedimentos auditivos, ceguera y muerte. En fetos en desarrollo, genera problemas de desarrollo motriz, problemas sensoriales y retardos mentales. El metilmercurio no se produce normalmente por los procesos industriales que utiliza este metal, sin embargo, el Mercurio liberado al ambiente, principalmente en cienos y lodos asociados a cuerpos de agua, puede sufrir la metilación por intermediación bacteriana en fase anaerobia. Una vez metilado, el catión es muy soluble y es fácilmente absorbido por peces y algunos invertebrados acuáticos donde se acumula en su tejido graso (Bishop, 2000).

El Mercurio elemental o metálico, es causante de efectos adversos a la salud cuando es inhalado en forma de vapor donde puede ser absorbido por los tejidos pulmonares. La exposición puede ocurrir cuando el Mercurio metálico se derrama, o productos que lo contengan se quiebran y exponen el elemento al aire, en especial cuando esto ocurre en climas cálidos o espacios interiores con mala ventilación. De igual forma, el calentamiento de amalgamas bajo condiciones de pobre recuperación, tiene el potencial de liberación de vapores de Mercurio metálico. La sintomatología derivada por intoxicación con este metal, es muy parecida a la que se observa por metilmercurio e incluye: temblores, cambios emocionales, como por ejemplo irritabilidad, nerviosismo, cambios de temperamento, timidez excesiva; también incluye insomnio, cambios neuromusculares tales como debilidad, atrofia muscular, espasmos; así mismo, se puede presentar dolor de cabeza, disturbios sensoriales, cambios en respuestas nerviosas y déficit de desempeño en pruebas de función cognitiva. A mayores exposiciones pueden ocurrir daños renales, fallas respiratorias y hasta la muerte. 
Los compuestos de Mercurio, tanto orgánicos como inorgánicos y en elevadas exposiciones como el Cloruro de Mercurio (II) o el Nitrato de Mercurio (II), pueden resultar en daños al tracto gastrointestinal, el sistema nervioso central y los riñones. Así mismo, los compuestos orgánicos como inorgánicos de Mercurio, son absorbidos por las mucosas gastrointestinales y llegan a otros sistemas a través de esta vía. Los compuestos orgánicos tienen mayor probabilidad de absorción por ingestión que los inorgánicos. Los síntomas asociados a altas exposiciones a Mercurio inorgánico incluyen irritación de la piel y dermatitis, pérdida de memoria, cambios de temperamento, disturbios mentales y debilidad muscular.

De estas formas de Mercurio, las dos primeras son las más comunes, siendo la primera normalmente indirecta, debido a que la ingesta de metilmercurio se hace a través del consumo de peces o moluscos donde esta sustancia se bioacumula (Varela Villegas \& Mateus Solarte, 2012).

La movilidad del Mercurio entre la fase acuosa y la fase sólida, depende del estado de valencia del elemento $\left(\mathrm{Hg}^{0}\right.$, $\mathrm{Hg}^{+} \circ \mathrm{Hg}^{+2}$ ), del nivel de $\mathrm{pH}$ del lugar y la concentración de ión cloruro $\left(\mathrm{Cl}^{-}\right)$, principalmente. El Mercurio se compleja muy fuertemente con el sulfuro (S-), pero este solo se encuentra en medios con condiciones reductoras. Reacciona con los cloruros en condiciones de $\mathrm{pH}$ ácido por encima de potenciales de óxidoreducción de $0.4 \mathrm{~V}$ para formar $\mathrm{HgCl}^{0}$. A pH por encima de 7, el complejo más común sería el $\mathrm{Hg}(\mathrm{OH})_{2}$. Inclusive, con la fuerte tendencia del Mercurio a generar complejos, la partición de estos en las fases no es significativa; la especie ionizada $\mathrm{Hg}^{2+}$ se encuentra fuertemente adherida a los minerales del suelo o sorbida sobre superficies inorgánicas y ligantes orgánicos. La tendencia del $\mathrm{Hg}^{2+}$ de fijarse sobre los suelos resulta en mínima migración (Watts, 1997). 
Las fracciones de Mercurio presentes en un entorno natural, se pueden dividir en tres grupos de acuerdo con su movilidad. La primera fracción, considerada la fracción móvil, corresponde con las especies orgánica (metilmercurio) y las sales de $\mathrm{Hg}^{2+}$. La fracción semimóvil se refiere principalmente a Mercurio elemental y amalgamas; y la fracción inmóvil se refiere a $\mathrm{HgS}$ y calomel $\left(\mathrm{Hg}_{2} \mathrm{Cl}_{2}\right)$ (Frentiu, Pintican, Butaciu, Mihaltan, Ponta \& Frentiu, 2013).

\section{Oro y Mercurio en Colombia}

U sando información referida por el "Boletín estadístico de minas y energía" de la UPME (Energía, 2014), la producción de metales preciosos en Colombia viene incrementándose, posiblemente por el aumento de la inversión extranjera que ha sido consistente en los últimos cuatro años en el país, pasando, para el caso del oro, a cerca de $15.000 \mathrm{Kg}$ en el primer trimestre del año 2013, un incremento superior al $10 \%$ con respecto al año anterior.

Según Casallas \& Martínez (2014), sobre la base de información de la Contraloría General de la República y la UPME, se afirma que en Colombia hay 17 departamentos y 80 municipios donde se llevan a cabo procesos de extracción artesanal, pequeña o industrial de oro; los departamentos de Antioquía y Bolívar poseen la mayor cantidad de minas del país, produciendo alrededor de 18.8 toneladas de oro anuales, si bien departamentos como Chocó, Córdoba, Caldas y Tolima también tienen amplia presencia de la actividad extractiva de oro. Las producciones de oro en toneladas, entre el año 2002 y 2013 se presentan a continuación (Tabla 1). 
Tabla 1. Producción de Oro (en Toneladas) en el periodo 2002-2013

\begin{tabular}{|l|c|}
\hline Departamento & Toneladas \\
\hline Antioquia & 177 \\
\hline Chocó & 84 \\
\hline Bolívar & 35 \\
\hline Caldas & 12 \\
\hline Cauca & 8 \\
\hline
\end{tabular}

Fuente. Elaboración propia de los autores, (2014), a partir de datos del Ministerio de Minas y Energía, (2013).

La actividad de extracción del oro en el país se podría nominar como atomizada, es decir, existen múltiples actividades de extracción en diferentes zonas de la región, la mayoría de las cuales no cuenta con una legalidad o formalización en su actividad. Según Casallas \& Martínez (2014), tomando datos oficiales, existen 4.133 unidades de minería que son equivalentes al $29 \%$ de la minería con o sin título minero, de las cuales 3.584 son ilegales. Lo anterior representa el $40 \%$ del total de la ilegalidad de minería en el país.

Por otra parte, de acuerdo con Varela \& Mateus (2012), la liberación de Mercurio al ambiente, asociada con la extracción primaria de metales en el país se resume a continuación (Tabla 2). 
Tabla 2. Liberaciones de Mercurio por subcategorías

\begin{tabular}{|c|c|c|c|c|c|c|c|c|c|}
\hline Categoria: Extracción & & & & & Liberac & jones es & imadas de $\mathrm{r}$ & $\mathrm{Hg}(\mathrm{Kg} \mathrm{Hg}$ & /año) \\
\hline Subcategoria & $\begin{array}{c}\text { Actividad } \\
\text { anual }\end{array}$ & $\begin{array}{l}\text { entrada } \\
\text { de } \mathrm{Hg} \\
\mathrm{g} / \mathrm{Ton}\end{array}$ & $\begin{array}{c}\text { de } \mathrm{Hg} \\
\text { (Kg/año) }\end{array}$ & Aire & Agua & Suelo & \begin{tabular}{|c|} 
Sub \\
productos \\
$\mathbf{e}$ \\
impurezas
\end{tabular} & $\begin{array}{l}\text { Residuo } \\
\text { s } \\
\text { general } \\
\text { es }\end{array}$ & $\begin{array}{l}\text { Tratamiento/ } \\
\text { disposición } \\
\text { residuos } \\
\text { espeófico por } \\
\text { sector }\end{array}$ \\
\hline $\begin{array}{l}\text { Producción de cobre a } \\
\text { partir de la mena } \\
\text { mineral }\end{array}$ & \begin{tabular}{|l|}
73214 Ton \\
mena \\
procesada/año
\end{tabular} & 17 & 1245 & 62 & 75 & 0 & 461 & 0,0 & 647 \\
\hline $\begin{array}{l}\text { Extracción de oro } \\
\text { mediante procesos } \\
\text { distintos de la } \\
\text { amalgamación con } \\
\text { mercurio }\end{array}$ & $\begin{array}{l}1270000 \text { Ton } \\
\text { de mena de } \\
\text { oro usado/año }\end{array}$ & 9 & 11430 & 457 & 229 & 10287 & 457 & 0,0 & 0,0 \\
\hline $\begin{array}{l}\text { Producción Primaria de } \\
\text { metales } \\
\text { ferrosos (hierro, acero) }\end{array}$ & \begin{tabular}{|l|}
280772 Ton \\
de hierro \\
producido/año
\end{tabular} & 0,1 & 14 & 13 & 0,0 & 0,0 & 0 & 0,0 & 1 \\
\hline $\begin{array}{l}\text { Extracción de oro } \\
\text { mediante } \\
\text { amalgamacón con } \\
\text { mercurio sin uso de } \\
\text { retortas }\end{array}$ & $\begin{array}{l}37,2 \text { Ton } \\
\text { de oro } \\
\text { producido/ } \\
\text { año }\end{array}$ & 4,2 & 157000 & 94200 & 31400 & 31400 & 0 & 0,0 & 0 \\
\hline $\begin{array}{l}\text { Producción de niquel a } \\
\text { partir de la mena } \\
\text { mineral }\end{array}$ & $\begin{array}{l}3600000 \text { Ton } \\
\text { mena } \\
\text { procesada/año }\end{array}$ & $<0,001$ & 3,6 & 0 & 0 & 0 & 0 & 0,1 & 0 \\
\hline
\end{tabular}

Fuente. Varela \& Mateus, (2012).

El Mercurio liberado al aire, eventualmente se sedimenta y hace parte de las otras dos fracciones. Sin embargo, al ser una liberación dispersa, sus efectos tienden a diluirse; pero de los valores dados anteriormente (Tabla 2), se puede observar que cerca del $37 \%$ liberado al ambiente, se encuentra en suelo y agua, siendo esta una cantidad alrededor de 62.8 ton/ año. En soluciones controladas en laboratorio, se ha logrado identificar que el Mercurio elemental puede llegar a valores de solubilidad de $1.99 \mu \mathrm{g} / \mathrm{l}$ (Yu, et al, 2011), sin embargo, la realidad de lo que ocurre en la interacción suelo-agua no es completamente conocida y por ello, lo que puede estar sucediendo en las riberas de los ríos donde se hace extracción de oro usando amalgamación con Mercurio, puede no solo ser un problema en términos de contaminación del recurso hídrico sino también del recurso suelo. 


\section{Remediación de sitios comprometidos con Mercurio}

e debe recordar que el Mercurio es un elemento químico, por lo que es imposible obtener su destrucción. Sin embargo, sí es posible modificar el comportamiento físicoquímico del elemento, ya sea modificando su estado de oxidación o controlando su movilidad. El primer caso comprende el uso de herramientas químicas, con el fin de reducir u oxidar el mercurio y lograr su acomplejamiento, o su precipitación. El segundo, involucra medios físicos de encapsulamiento o atrapamiento (solidificación/estabilización o vitrificación), donde el Mercurio simplemente es <<atrapado>> en una matriz sólida que impide su contacto y movimiento hacia el entorno de acuerdo con la Agencia de Protección Ambiental de los Estados Unidos (USEPA, 2007). Los fenómenos de sorción (adsorción y absorción), también son utilizados para la remoción de Mercurio de ciertos ambientes; sin embargo, este tipo de medio físico trae como consecuencia la migración de la sustancia y no necesariamente su inmovilización.

Basándose en resultados de la aplicación de diversas metodologías de remediación, se reporta que cuatro metodologías de remediación son mayormente utilizadas para remediaciones en fase sólida, para suelos y residuos, y cuatro metodologías utilizadas para remediaciones en fase líquida para aguas (USEPA, 2007). Cabe destacar que solamente la metodología de solidificación/ estabilización ha sido utilizada para el tratamiento de mercurio elemental, todas las demás han sido utilizadas con compuestos mercuriados. 


\subsection{Mecanismos para fase sólida}

Para la fase sólida se han identificado como metodologías de remediación aplicables la solidificación/estabilización, el lavado de suelo (Soil Washing), tratamiento térmico y vitrificación.

El proceso de solidificación liga el metal a un bloque sólidos de material donde queda atrapado. Este bloque es, a su vez, menos permeable al agua que el suelo mismo. La estabilización que se genera a través de una reacción química, hace que el metal sea menos susceptible de ser lixiviado al ambiente.

El lavado de suelo consiste en un proceso mecánico que usa líquidos, generalmente agua, algunas veces aditivada, para depurar los suelos.; este proceso remueve los metales y los concentra en un volumen menor. Los metales tienden a estar ligados o adsorbidos, ya sea química o físicamente, a las partículas de limos o arcillas; estas últimas, a su vez, tienden a unirse a las partículas de arena o grava. El proceso de lavado de suelo, separa las partículas finas contaminadas (arcillas y limos) de las partículas gruesas (arenas y gravas). Una vez se termina el proceso, un menor volumen de suelo, que contiene la mayoría de las partículas finas, puede ser tratado por otros métodos (p. e. incineración o biorremediación) o dispuesto de acuerdo con la normatividad vigente. La mayoría del volumen de suelo residual se considera limpio.

El quemado y condensado (Roasting/Retorting) e incineración, son las técnicas de tratamiento térmico más utilizadas aplicadas al material contaminado con Mercurio. La primera operación separa el Mercurio del resto del material y lo condensa para su recuperación o remoción; la segunda, lo vaporiza y este debe ser recuperado en los sistemas de control de emisiones. 
La vitrificación se considera como una tecnología probada para la disposición y almacenamiento a largo plazo de materiales radiactivos, normalemente metales, el cual puede ser usado en metales no radiactivos. El proceso se conoce también como geofundición y se puede llevar a cabo in-situ o ex-situ. El primero, se lleva a cabo en el lugar donde se encuentra el propio suelo comprometido, a través del uso de electrodos que se entierran en el lugar y funden el material suelo, que al enfriarse genera una cápsula vítrea que contiene el metal. La técnica ex-situ, implica la remoción del suelo impactado y la introducción de este entre un horno donde se funde y se crea el material sólido. El material resultante es similar a la obsidiana y no permite la lixiviación de los metales que queden atrapados en su matriz.

Otros métodos, como el uso de plantas para la captura de metales pesados, el uso de plantas y sustancias quelantes (She, et al., 2014), o incluso el uso de nanotecnología para mejorar los procesos de remediación (Cameselle, et al., 2013), son parte de los estudios que se vienen desarrollando en múltiples centros de investigación alrededor del mundo en la actualidad, y en donde hay gran potencial para realizar desarrollos en los próximos años.

\subsection{Mecanismos para la fase líquida}

Para la fase líquida se tienen identificadas la precipitación/ coprecipitación, adsorción, filtración por membranas y la biorremediación como técnicas útiles para la remediación.

La técnica de precipitación/coprecipitación, comprende la remoción del metal de solución ya sea de forma directa a través de una reacción química que genere una especie insoluble, o indirecta, cuando el metal es removido por 
arrastre tras la precipitación de otra sustancia. Esta técnica es bastante utilizada cuando se tienen grandes volúmenes de agua comprometida.

La adsorción de Mercurio como forma de remoción en aguas, implica la adhesión del mismo a la superficie sólida de un material afín -p. e. carbón activado, arcillas modificadas). Esta tecnología tiene muchos limitantes cuando el Mercurio se encuentra presente en conjunto con más sustancias y se utiliza preferiblemente en pequeños sistemas o como técnica de pulimento final del efluente de otras tecnologías de tratamiento.

La remoción de Mercurio a través de membranas, se realiza por retención física del mismo, ya que la membrana impide su paso a través de ella. Esta tecnología presenta una gran desventaja ya que, no obstante, al producir un efluente de gran calidad, el Mercurio queda en una mayor concentración en una fracción del volumen de agua denominada rechazo, que requiere de un tratamiento posterior.

Los procesos de biorremediación involucran la intermediación bioquímica, ya sea por retención dentro de la biomasa (She, et al., 2014), o modificación de la especiación de Mercurio para removerlo de la masa de agua.

El desarrollo de nuevas técnicas o la refinación y comprensión de la fenomenología de las técnicas más utilizadas, es el centro del estado del arte en la actualidad. 


\section{Limitaciones para la aplicación de remediación de suelos en Colombia}

ualquier proceso de remediación de suelos requiere de unas etapas que, sobre la base del reconocimiento de la información disponible, utilizan métodos que permiten reconocer la magnitud del problema y los posibles factores magnificadores o minimizadores de la situación, permitiendo así tener una dimensión de realidad antes de iniciar cualquier tipo de tratamiento.

De la recopilación de esta información, los técnicos pueden plantear alternativas de recuperación que estén vinculadas con las características de la zona, las posibilidades tecnológicas y económicas, la disponibilidad, el potencial riesgo generado e incluso, la necesidad de control por factores especiales -peligrosidad del material o probabilidad de contacto con ecosistemas estratégicos o seres humanos, por poner algunos ejemplos-, como se menciona en Martínez (2013).

De esa forma, claramente se observa que entre mayor sea la disponibilidad de información sobre el incidente y las condiciones donde ocurre, las decisiones técnicas pueden ser más efectivas y eficientes; sin embargo, en Colombia se carece de gran detalle de información sobre el mercurio y sus condiciones de uso en distintas partes del país, pues se trata de una actividad sin un control suficiente que requiere vigilancia estricta y permanente (Procuraduría Nacional de Nación, 2011).

Lo atomizado de la actividad minera del oro, que en la mayoría de los casos obedece a intereses que buscan generar un lucro fácil a costa de riesgos para la salud de las personas y el ambiente, en sitios de difícil acceso en donde las propias condiciones hacen complicado el arribo de personal o equipos, 
genera que en la actualidad solo se conozcan las situaciones en donde la degradación ambiental es muy grande y resulta evidente a través de la modificación de cauces de ríos, tala de árboles o en el daño a la superficie terrestre, como quiera que la minería carente de control implica un significativo riesgo de erosión (Procuraduría Nacional de Nación, 2011).

Se debe analizar que la contaminación de la matriz suelo y la matriz agua por los excesos de Mercurio usados durante el proceso de amalgamación, no es observable sino a través de métodos analíticos de laboratorio, y que sus consecuencias son acumulables en el tiempo; por ello, normalmente no se evidencian en el corto plazo. Por lo anterior, es claro que el problema reviste de una amplia importancia, pero así mis-mo, requiere de amplios recursos para poder analizar esos impactos ambientales negativos no evidentes, que pueden tener consecuencias catastróficas para la salud humana y del ecosistema.

Las actividades de remediación en dichas regiones deberían ser prioritarias; sin embargo, al no tener una persona jurídica o natural que pueda responder por los daños, configura una situación en donde el Estado tendría que hacerse cargo de reparar los daños generados en los ecosistemas, lo cual termina siendo lesivo para las finanzas públicas por el estado de minas abandonadas y los costos asociados a la recuperación de la base natural impactada (Procuraduría Nacional de Nación, 2011).

De esta forma, en el país es absolutamente necesario el desarrollo de investigaciones que contribuyan a esclarecer el panorama técnico de la situación de estos sitios donde se viene usando el Mercurio a través de malas prácticas, bien en la matriz agua, en la biodiversidad y cadenas tróficas, en el aire o en el suelo. Estas investigaciones acopiarán la información que es necesaria para identificar la problemática de forma adecuada, así como viabilizar las mejores alternativas de remediación, al menos desde las pruebas de laboratorio, con miras a adaptar las tecnologías que permitan optimizar los recursos necesarios para atender las problemáticas. 


\section{Conclusiones}

e entiende que las condiciones socio-económicas asociadas históricamente con la explotación minera artesanal y desafortunadamente la ilegal en épocas más recientes, se encuentran asociadas con la utilización del Mercurio, insumo cuyo deficiente manejo ha traído como consecuencia afectaciones ambientales que dada su condición, ubicación y modo de ocurrencia son muy difíciles de intervenir y asignar responsables.

El Mercurio y los compuestos orgánicos e inorgánicos que se usan o se generan de forma espontánea en condiciones naturales, tienen un comportamiento que se desconoce parcialmente en términos de la realidad colombiana, por lo que se hace necesario desarrollar sistemáticamente investigaciones sobre su estabilidad en medios acuosos, en la matriz suelo e incluso en el aire, con el fin de determinar el riesgo por exposición al que pueden estar sometidas las áreas en las cuales se realiza explotación del oro usando el método de amalgamación.

Las empresas que se encargan de la explotación del oro, deben propender por la formalización y de paso, por la aplicación de mejores prácticas en el beneficio del mineral precioso, implementando mejores prácticas y procesos que contribuyan con la disminución del riesgo por exposición a sustancias tóxicas por parte de empleados, comunidad y el mismo ecosistema; ahora bien, para el caso de extracción ilegal del mineral, es necesario que los procesos de control y vigilancia sean efectivos, porque más allá del no pago de regalías y las condiciones inadecuadas de trabajo, los problemas ambientales y sanitarios asociados pueden llegar a ser de alta relevancia en ciertas zonas. 
Existen múltiples tecnologías que permiten el desarrollo de procesos de remediación en fase acuosa y en fase sólida; por ello, resulta importante avanzar en el conocimiento de la problemática para lograr adaptar las tecnologías a las condiciones de las zonas de explotación, y de esa forma, permitir que no existan limitantes técnicos o económicos que impidan que se haga una correcta descontaminación de suelos contaminados con Mercurio.

La acción del Estado se encuentra muy restringida, tanto desde el punto de vista logístico, como legal y técnico, para poder acometer las acciones diagnósticas y remediales acorde con el estado del arte. Es por esta razón que la determinación de alternativas de tratamiento se vuelve una necesidad primordial que el trabajo de investigación que se realiza en la Universidad EAN, así como otros trabajos en otros centros de investigación, pretenden apropiar como sus objetivos últimos. 


\section{Referencias bibliográficas}

Bishop, P. L. (2000). Pollution Prevention: Fundamentals and Practice, New York, NY, USA: McGraw Hill.

Boening, D.W. (1999). Ecological Effects, Transport, and Fate of Mercury: A General Review. Chemosphere 40, pp. 13351351.

Cameselle, C., Reddy, K.R., Darko-Kagya, K., \& Khodadoust, A. (2013). Effect of Dispersant on Transport of Nanoscale Iron Particles in Soils: Zeta Potential Measurements and Column Experiments. Journal of Environmental Engineering, 139(1), pp. 23-33. American Society of Civil Engineers.

Casallas, M., \& Martínez S., J. A. (2015). Panorama de la minería del oro en Colombia. Working Paper. Universidad EAN.

Energía, M. d. M. y. (2014). Boletín Estadístico de Minas y Energía 2008-2012. U. d. P. M. Energética, ed., 239.

Frentiu, T., Pintican, B. P., Butaciu, S., Mihaltan, A. I., Ponta, M., \& Frentiu, M. (2013). Determination, speciation and distribution of mercury in soil in the surroundings of a former chlor-alkali plant: assessment of sequential extraction procedure and analytical technique. Chemistry Central journal, 7(1), p. 178.

Henry, J.R. (2000) An Overview of the Phytoremediation of Lead and Mercury, Report prepared for U.S. Environmental Protection Agency Office of Solid Waste and Emergency Response Technology Innovation office. Washington, D.C. 
Martínez, J.A. (2013). Memorias de unidad: Estudio de Impacto Ambiental. Especialización en Gestión de Residuos Sólidos. Universidad EAN.

Martínez, J.A. \& Uribe, A. (2014). Remediación de suelos contaminados a través de tratamientos no convencionales. Informe de avance de investigación - Vicerrectoría de Investigación, Universidad EAN.

Procuraduría Nacional de la República (2011). Minería ilegal en Colombia: informe preventivo. Procuraduría Nacional de la República, Bogotá. 2011. Recuperado de: http:// www.procuraduria.gov.co/portal/media/file/MINERIA\%20 ILEGAL\%20EN\%20COLOMBIA\%20\%20DOCUMENTO.pdf

She, W., Cui, G., Jie, Y., Bai, Y., Cao, Y., \& Xiao, C. (2014). Comparative effects of chelants on plant growth, cadmium uptake and accumulation in nine cultivars of Ramie (Boehmeria nivea ). Acta Agriculturae Scandinavica: Section B, Soil \& Plant Science, 64(1), 71-78. doi:10.1080/09064710.2014.88 5077.

USEPA (2007). Treatment Technologies For Mercury in Soil, Waste, and Water U.S. Environmental Protection Agency Office of Superfund Remediation and Technology Innovation, Washington, DC.

Varela Villegas, A., \& Mateus Solarte, J. C. (2012). Evidencia científica, normativa y técnica sobre la problemática del mercurio en Colombia - Revisión sistemática de la literatura. Ministerio de Salud y Protección Social - Fundación para la Educación y el Desarrollo Social FES, 105.

Wang C, Zhu D, YI F, et al. (s.f). Soil Quality Evaluation and Technology Research on Improving Land Capability -- A Case Study on Huanghuaihai: Plain in Shandong Province. 
Agricultural Science \& Technology [serial online]. November 2014;15(11), pp. 1960-1966. Available from: Academic Search Elite, Ipswich, MA.

Watts, R. J. (1997). Hazardous Wastes: Sources, Pathways and Receptors. New York, NY, USA: Wiley.

Yu, V. Alekhin, N.R. Zagrtdenov \& R.V. Mukhamadiyarova. (2011). $\mathrm{Hg}^{\circ}$ (liq)- $\mathrm{Hg}^{\circ}$ (solution) Equilibrium and solubility of elementary mercury in water. Moscow University Geology Bulletin, 66(6), pp. 439-441. Moscow: Allertos Press, Inc. 